C-A/AP/\#194 March 2005

\title{
RHIC abort thyratrons with new trigger modules
}

\author{
Harald Hahn, Yugang Tan, David Warburton
}

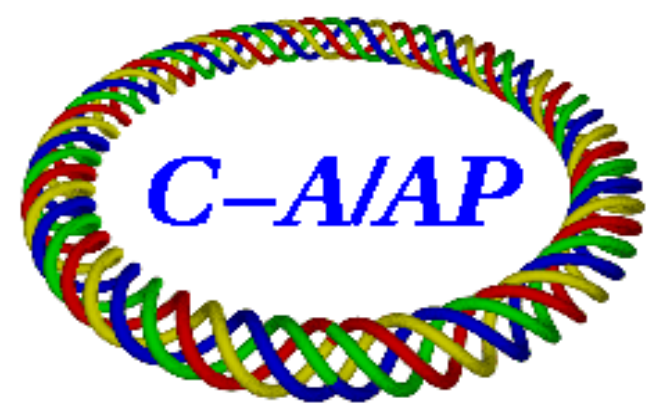

Collider-Accelerator Department Brookhaven National Laboratory Upton, NY 11973 


\title{
RHIC abort thyratrons with new trigger modules
}

\author{
Harald Hahn, Yugang Tan, and David Warburton
}

\section{INTRODUCTION}

Already during its development phase, the RHIC abort kicker system was prone to prefire and could not reliably hold the "design" voltage of $33 \mathrm{kV}$ for the nominally required 10 hour storage time. Even at the reduced $27 \mathrm{kV}$ for the operational runs the system experienced pre-fire problems. Several changes were implemented by the Pulsed Power group for the 2004 run and the frequency of pre-fires has been significantly reduced. Ongoing efforts are directed at further increasing the reliability of the abort systems. Results from our studies to develop a better understanding of the thyratron switch tubes are presented here. All tests were done with a low-value discharge capacitor unit to prevent damage to the tubes.

Two types of thyratron discharge tubes are in use, the CX1575C in the blue ring and the CX3575 in the yellow ring, and also in the blue ring for the 2005 run when a replacement is required. A different tube type, a deuterium filled gas discharge tube HX3002 has been considered as a possible future pulse switch in the abort kickers. The output of the trigger module presently being used for abort kickers does not have enough power to trigger the gas tube, and their chassis are obsolete and will be replaced by VME enclosures. A new trigger module, satisfying both objectives, has been designed by Yakov Kotlyar, and units in sufficient quantity for both rings have been manufactured. The new modules with new HV isolation transformers are being considered for installation after further reliability testing. The modules generate better than $1 \mathrm{kV}$ into $50 \Omega$ with a rise time of $\leq 50 \mathrm{~ns}$. Results from the study of the impact of the new trigger module on the thyratron operation are presented in this note. 


\section{TRIGGER CIRCUITS}

The thyratrons in the RHIC abort systems are all discharge-triggered using the vendor approved DC-primed circuit, shown in Fig. 1 (left). The DC-primed circuit offers continuous DC current to grid-1, and is ready for the discharge trigger on grid-2. The size of the prime current, which is controlled by the fixed prime voltage of unloaded $\sim 150 \mathrm{~V}$ and the resistor R1, presently $100 \Omega$ in blue and $250 \Omega$ in yellow, determines to some degree the required trigger voltage at injection, $\sim 4 \mathrm{kV}$. The grid -1 resistors in the $\mathrm{CX}$ 3775 of Yellow were changed from $100 \Omega$ to $250 \Omega$ for the Run 2004 in order to keep the grid-1 current within the recommended range from 150 to $300 \mathrm{~mA}$. In fact, the run had fewer pre-fires and although there were other changes, the improvement was attributed primarily to the resistor change. Thus, the primary objective of the ongoing R\&D was to demonstrate the correlation between grid-1 current and high-voltage holding, and by implication the sensitivity to pre-fire. The expectation was that voltageholding capability is improved by reducing the grid-1 current while holding the reservoir voltage at the "stamped-on" voltage.

An alternate system, the Split-trigger circuit in Fig. 1 (right), is used for the thyratron of the g-2 Quadrupole Pulser in the AGS. This circuit has no prime current and is shown to be less prone to pre-fire. A strong trigger pulse is required to draw current from grid \#1 prior to the delayed grid \#2 trigger current. This circuit simulates the standard twotrigger ("black box") system used on the RHIC injection thyratrons, but which cannot be implemented due to the floating cathode of the abort thyratron.
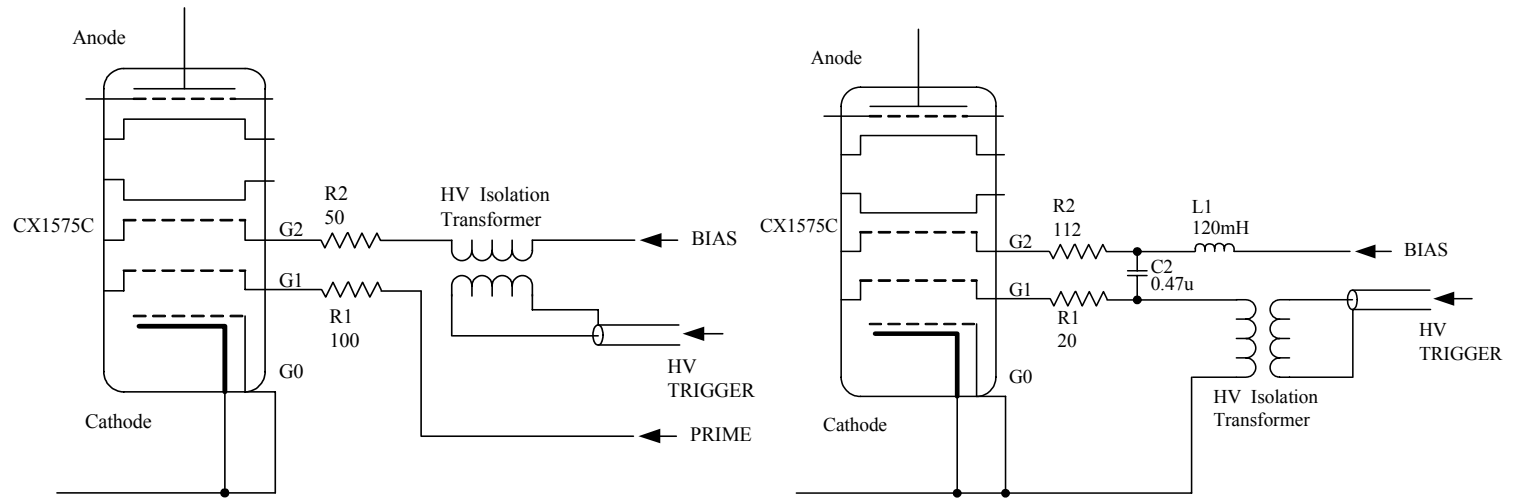

Fig. 1. Trigger systems, DC-Primed (left) and Split-Trigger (right) 


\section{Trigger Voltages in DC-primed circuit}

The voltage at grid $\# 2$ of the DC-primed thyratron, CX1575C S/N 1516, the thyratron type used in the blue ring, generated by the new trigger module is shown in Fig.2 (note the 100:1 voltage divider). The measurements are performed on the thyratron with $R_{1}=100 \Omega$, corresponding to a prime current in grid-1 of $\sim 450 \mathrm{~mA}$, but without anode voltage and current. The reservoir voltage had to be set above the stamped-on value of $4.5 \mathrm{~V}$ to allow discharge at $4 \mathrm{kV}$ anode. The new trigger module produces a fast $\sim 1 \mathrm{kV}$ pulse which arrives at the grid- 2 after a delay of $\sim 300 \mathrm{~ns}$, which is caused by the cable between trigger unit and thyratron in the ring.

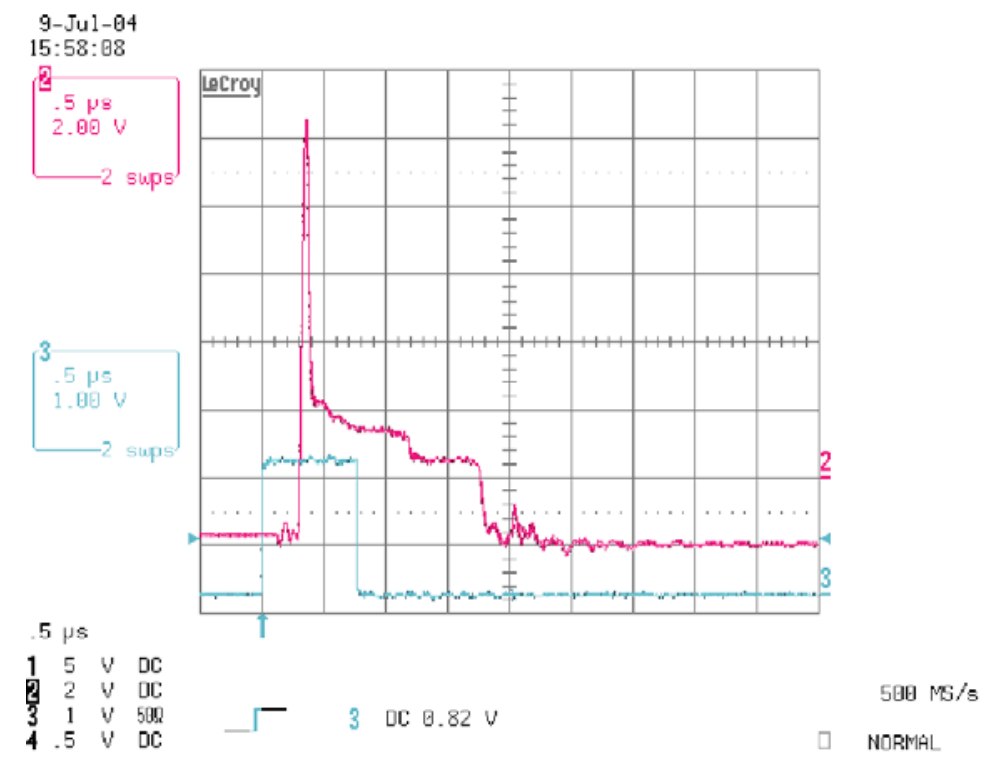

Fig. 2. Grid 2 voltage (red, trace 2), New Trigger Module;

(DC Prime $\sim 1 \mathrm{~A}, \mathrm{U}_{\mathrm{RE}}=5.30 \mathrm{~V}$ )

The grid-2 voltages generated by the new and old trigger module are compared in Fig.3. The thyratron is DC-primed with a grid-1 resistor of $100 \Omega$. The rise time of the new module is $\sim 50$ ns versus $\sim 200 \mathrm{~ns}$ in the old one, and the peak voltage, before the grid draws current, is $1.1 \mathrm{kV}$ versus $400 \mathrm{~V}$. Full grid-2 current (i.e. grid-2 voltage $\sim 400 \mathrm{~V}$ ) is reached after $\sim 120$ ns with the new versus $\sim 250$ ns with the old module. Note the jitter in the grid- 2 current,$\sim 10 \mathrm{~ns}$ versus $\sim 40 \mathrm{~ns}$, which is a property of the thyratron that depends on the trigger module voltage rise time and is reduced with the new module. 

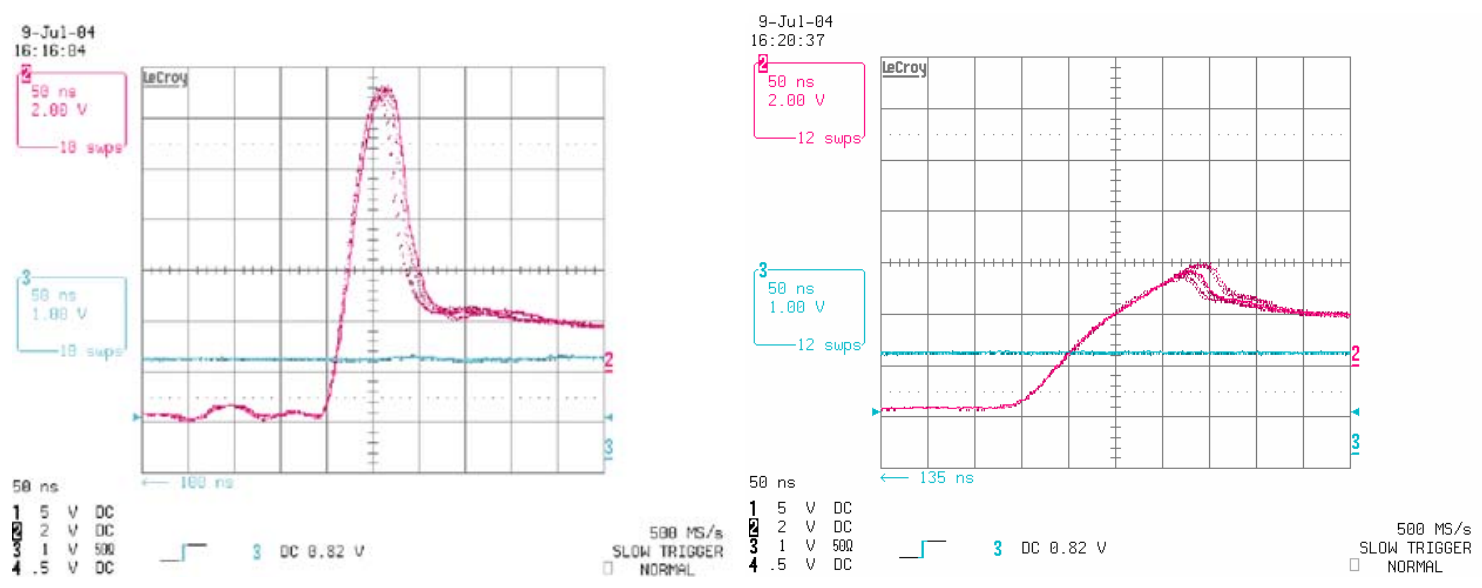

Fig.3. Grid 2 voltage with the new (left) and old (right) trigger module.

The dependence of the grid-2 voltage response on the grid-1 prime current is demonstrated in Fig.4 measured with the new module. The full current corresponding to $\sim 450 \mathrm{~V}$ is reached after $\sim 150 \mathrm{~ns}$ for $R_{1}=500 \Omega\left(I_{1} \approx 200 \mathrm{~mA}\right)$ and after $\sim 200 \mathrm{~ns}$ for 1 $\mathrm{k} \Omega\left(I_{1} \approx 120 \mathrm{~mA}\right)$. At the start of this study, it was assumed that smaller grid-1 currents make the thyratron more difficult to trigger implying greater resistance to pre-fire.

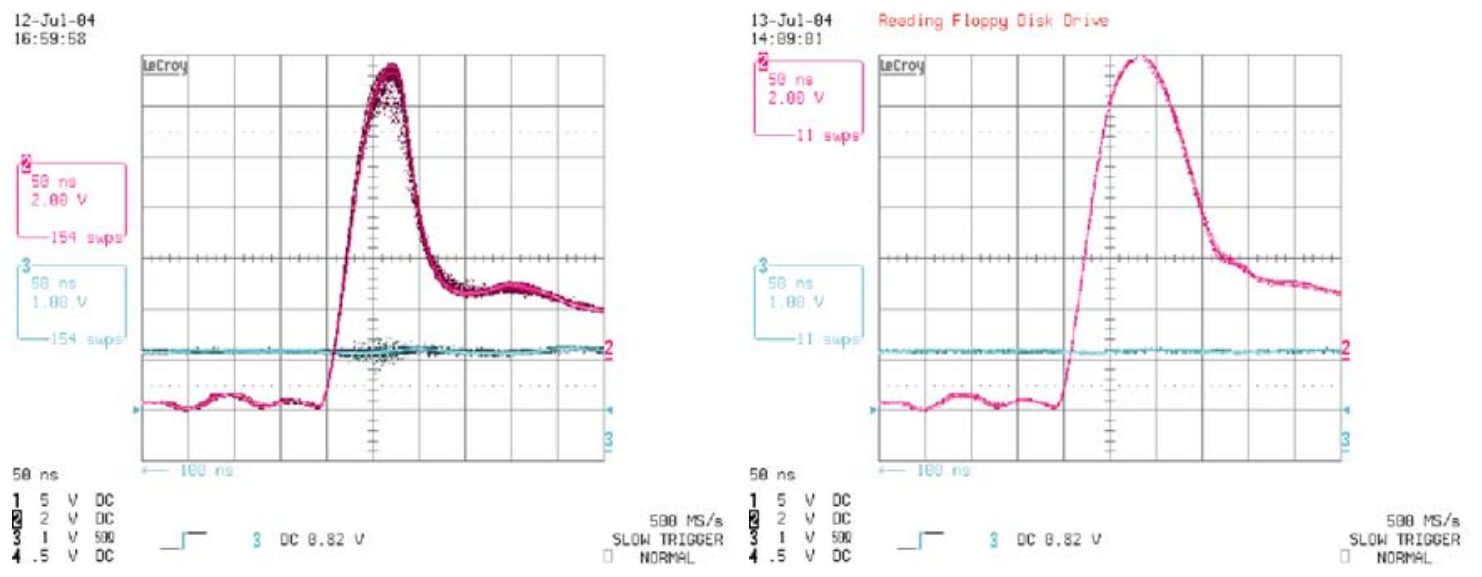

Fig.4. Dependence of grid-2 voltage on grid-1 resistor, left $500 \Omega$, right $1 \mathrm{k} \Omega$.

\section{Trigger Voltages in split-trigger circuit}

The grid voltages generated by the new module with the split trigger circuit are shown in Fig. 5. At the left, one sees grid-1 voltages, both directly at the grid and at R 1 which also drives grid-2. The grid-2 voltage with the negative bias is shown on the right side of Fig. 5 with expanded time scale. Full grid-2 current is reached only after $\sim 250 \mathrm{~ns}$. 

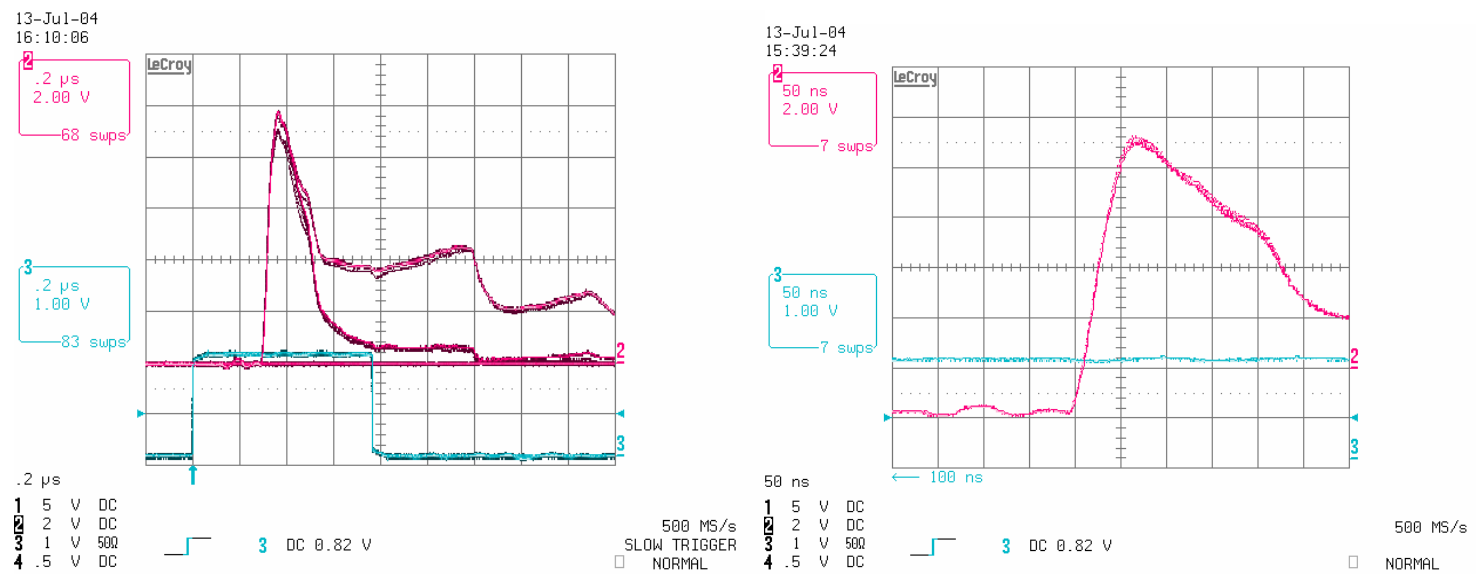

Fig.5. Voltages at Grid $1 \&$ R1 (left) and grid-2 (right) with split-trigger circuit.

\section{ANODE CURRENT DELAY at $4 \mathrm{kV}$}

The RHIC beam must be reliably aborted from injection to storage at full energy, corresponding to thyratron voltages from $\sim 4 \mathrm{kV}$ to $>27 \mathrm{kV}$. The beam bunch circulates the ring in $12.8 \mu \mathrm{s}$. A $4 \mu$ s gap in the beam is provided to allow for a pulse rise time of $\sim 1 \mu \mathrm{s}$, accommodating timing errors plus the delay time of a redundant trigger in case of thyratron pre-fire. The cable between trigger module in the service room and the ring results in an inconsequential $\sim 0.3 \mu$ s delay. The delay in the thyratron discharge depends on the anode voltage, the trigger circuitry, the reservoir voltage and strongly on the grid-1 current . The anode discharge delay in the two thyratron types was here measured as function of reservoir current and converted by means of a calibration run to reservoir voltage. The curves were taken at constant anode voltage of $4 \mathrm{kV}$ with the grid- 1 resistor as variable parameter. Note however that the anode delay time is not by itself the concern here, rather it is studied as an indicator of thyratron stability in search of voltage holding capability.

\section{Thyratron CX1575C}

All measurements for the CX1575C thyratrons were made on the tube with SN 1516 with has stamped-on reservoir voltage of $4.5 \mathrm{~V}$. The "stamped-on" reservoir voltage is determined at the factory to assure a 1 hour holding voltage of $50 \mathrm{kV}$ and is obtained with

a prime current of typically $100 \mathrm{~mA}$ and a negative grid-2 bias of $\sim 100 \mathrm{~V}$. The thyratrons 
do not discharge at $4 \mathrm{kV}$ when set to the stamped-on reservoir voltage, and the tubes in the blue ring run all at higher reservoir voltages.

The discharge delay in the thyratron was measured with the split-trigger and dcprimed circuits. The data are shown in Fig. 6. Clearly, the split trigger circuit has a greater delay of $\sim 0.2 \mu \mathrm{s}$, but note the wider range of reservoir voltages allowing operation at lower reservoir voltage with the resulting increased stability against pre-fires.
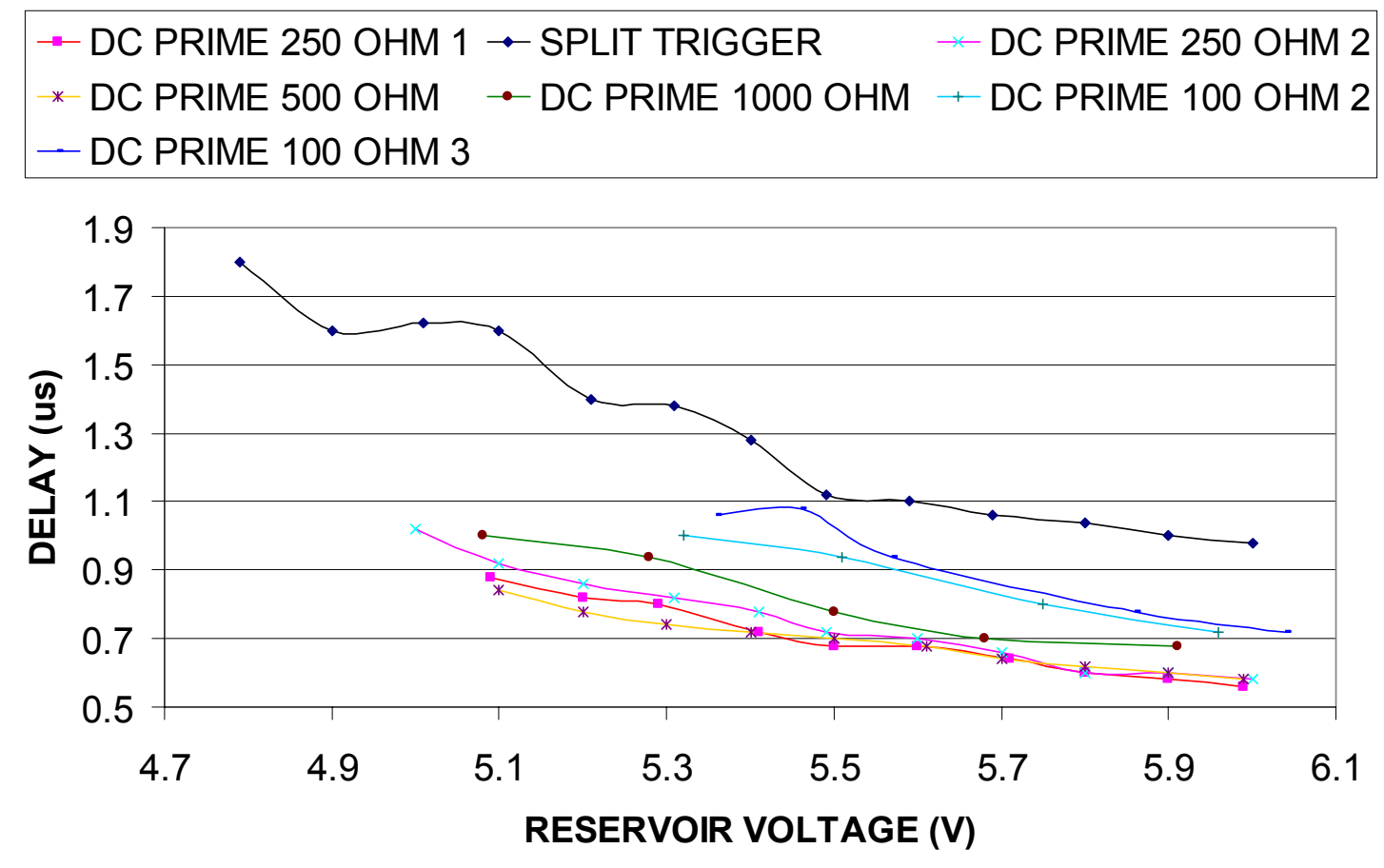

Fig. 6. Discharge delay versus reservoir voltage in the CX1575C.

TheCX1575C thyratrons in the blue ring are runing with a $100 \Omega$ grid-1 resistor leading to $\sim 450 \mathrm{~mA}$ current, a value quite different from the vendor test conditions. The grid-1 current is largest with $100 \Omega$ and reduced to 300,177 , and $80 \mathrm{~mA}$ with 250 , 500 , and $1000 \Omega$. Note that the tube has been factory tested at $100 \mathrm{~mA}$, achievable with $\sim 800 \Omega$. The delay for the DC Prime with $250 \Omega$ was measured twice, the first run showed significant jitter ( $0.5 \mu$ s at the low voltage end) but was quiet the second day. The reservoir voltage at which the tube did not reliably discharge was 5.0 and $4.9 \mathrm{~V}$ in these runs. Overall, a certain amount of jitter in the results was observed. 
The expectation was that the higher grid-1 current would reduce the trigger threshold. Somewhat puzzling is thus the larger anode current delay with the $100 \Omega$ grid-1 resistor in the DC primed trigger circuit. It seems that the delay goes through a minimum at 250 to $500 \Omega$ and increases again with increasing grid-1 resistors (or decreasing currents) as indicated by the $1000 \Omega$ curve. One can speculate that a shorter delay indicates an increased sensitivity to the grid-2 trigger voltage (and other possible noise sources responsible for pre-fires). Although plausible and supported by the subsequent data for the $\mathrm{CX} 3775 \mathrm{C}$ thyratron, no experimental confirmation was attempted.

\section{Thyratron CX3575C}

Anode current delay measurements were extended to the thyratron tube type CX3575C, which was recommended by the vendor to minimized the anode damage due to the significant reverse current, and which is used in the yellow ring and now also in the blue ring when replacements are required. The stamped-on reservoir voltage for this tube type is obtained at the lowered $40 \mathrm{kV}$ anode voltage and with a grid- 1 current of $250 \mathrm{~mA}$. The results for the CX3575C ( Serial \# 1774, with the stamped-on Ures $=5.4 \mathrm{~V}$ ) are shown in Fig. 7. All measurements were here made with the dc-prime circuit and the new trigger unit.

The most visible difference of this tube is the lower reservoir voltage $(\sim 0.5 \mathrm{~V})$ required to trigger at $4 \mathrm{kV}$. The measurements of this tube focused on the delay jitter as function of reservoir voltage. The figure shows the jitter range by using two equally colored graphs. It is noted that the $100 \Omega$ grid-1 resistor with a $\sim 450 \mathrm{~mA}$ prime current has a noticeable longer delay and larger jitter compared to the 250 and even more so to the 500 $\Omega$ resistors. In the rings, the tubes are operated with $250 \Omega$ grid-1 resistors and $\sim 280 \mathrm{~mA}$ prime current. The vendor data sheet suggests a grid-1 range from 150 to $300 \mathrm{~mA}$, obtained with the 250 or $500 \Omega$ resistor. The $1 \mathrm{k} \Omega$ resister allows triggering at $4 \mathrm{kV}$ with much lowered reservoir voltages (perhaps indicating enhanced sensitivity to prefire). The discharge delay at higher anode voltages is essentially independent of the grid-1 resistor. 
Tests were also made with 5 and $10 \mathrm{k} \Omega$ resistor, but the delay jitter became excessive and the curves are not shown.

CX3575C Delay @ 4 kV

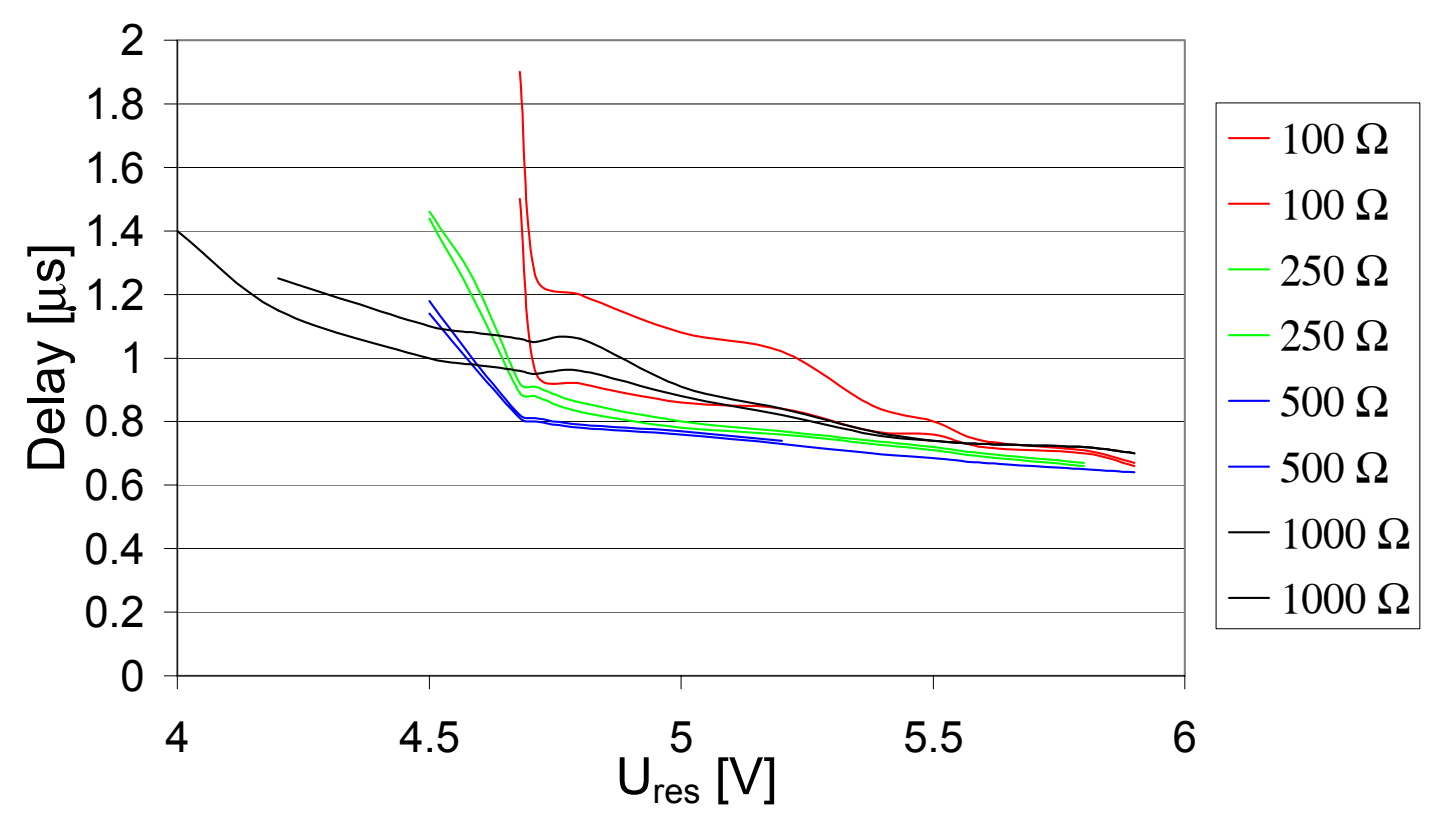

Fig. 6. Discharge delay versus reservoir voltage in the CX35575C.

\section{HIGH-VOLTAGE HOLDING TESTS}

The maximum (i.e. stamped on) reservoir voltage for the nominal anode voltage is obtained at the vendor by holding $40 \mathrm{kV}$ voltage for 1 hour. This procedure was applied to the CX3575C (S.N. 1774) in order to establish a correlation between different grid-1 resistors and voltage holding capability. The test was extended to the split trigger circuit with $\mathrm{R} 1=20 \Omega$. The results are shown in Table 1 . It was noted that the difference between 1 hour stable and a discharge within a few minutes can be produced by $50 \mathrm{mV}$ reservoir voltage change. Consequently, the voltages quoted can have a $\pm 0.05 \mathrm{~V}$ error. Nevertheless, the results suggest that the $\mathrm{R} 1=100 \Omega$ is most stable which seems to be at variance with our previous assumptions. 
Table 1. Voltage Holding in CX3575C at various grid-1 resistors

\begin{tabular}{cccc}
\hline R1 $[\Omega])$ & I1 $[\mathrm{mA}]$ & Ires [A] & Ures [V] \\
100 & 450 & 9.75 & 5.3 \\
250 & 280 & 9.7 & 5.25 \\
500 & 200 & 9.65 & 5.2 \\
$1 \mathrm{k}$ & 120 & 9.7 & 5.25 \\
$5 \mathrm{k}$ & 26 & 9.7 & 5.25 \\
$10 \mathrm{k}$ & 15 & 9.7 & 5.25 \\
Split Trggr & 0 & 9.65 & 5.2 \\
\hline
\end{tabular}

\section{DISCUSSION}

Reducing the reservoir voltage is the vendor-recommended method to avoid or minimize pre-fires. The counter indication to lowering the reservoir voltage is the need to provide adequate gas density in the tube and prevent "white arcs" due to ion currents at voltage levels above $\sim 30 \mathrm{kV}$. [Hahn \& Dunbar, AD/RHIC/RD -127 (1999)]. The Beam dump system with all 5 thyratrons active requires only $\sim 27 \mathrm{kV}$, and tolerates operation with lowered reservoir voltages. Balancing the requirements of holding high anode voltage on one hand and avoiding "starved electron" anode currents on the other hand determines the optimum choice of the reservoir voltage. The experimental observations are interpreted by a thyratron model, in which the overall deuterium gas density is determined by the reservoir voltage. The electron or ionized gas density between cathode and grid-2, which determines the trigger sensitivity, is given by the prime current and thus the grid -1 resistor. The present study was undertaken to test if changes in the trigger circuit or of the prime current intensity would allow stable operation without reduced reservoir voltage. The present results show a small but not decisive correlation. On the other hand, in case of repeated pre fires, a reduction of the reservoir voltage by only $0.05 \mathrm{~V}$ can significantly improve stability without danger of producing "white arcs". 
The stamped-on reservoir voltage for the CX3575C is determined with $40 \mathrm{kV}$, resulting in typically higher reservoir voltage values. The Yellow ring had the CX3575C thyratrons from the beginning. The original $100 \Omega$ grid-1 resistor was replaced in the 2004 run with $250 \Omega$ to bring the current close to the vendor test value of $250 \mathrm{~mA}$ and into the data-sheet specified range of 100 to $300 \mathrm{~mA}$ range. The measurements presented here do not support the opinion that this change resulted in an improved voltage holding capability. Reducing the grid-1 resistor value again to $100 \Omega$ could be considered after further tests. Lowering the reservoir voltage by $0.05 \mathrm{~V}$ remains the primary option if prefire problems reappear.

The specified anode voltage for the CX1575C thyratrons in the blue ring was $50 \mathrm{kV}$ and the reservoir voltage was appropriately lowered for this value, but with the consequence of having to run the reservoir above the stamped-on value for RHIC injection. For unknown historical reasons, these tube have a $100 \Omega$ grid-1 resistors resulting in a prime current of at $\sim 50 \mathrm{~mA}$, well above the $100 \mathrm{~mA}$ test value. For the 05 run, as of today, one of the CX1575C tubes in Blue has been replaced by a CX3575C with a $250 \Omega$ while retaining the $100 \Omega$ grid-1 value in the remaining tubes. No voltage holding measurements were made directly on the CX1575C tubes an the results from the other thyratron type hardly suggest a gain from changing the grid -1 resistor. Only lowering of the reservoir voltage can be suggested considered if pre-fire problems reappear.

The present study confirms the suitability of the new trigger circuit for the thyratrons in use, assuming that the long-term reliability has been demonstrated. The units are more than adequate to trigger the thyratrons with any grid-1 resistor chosen.

\section{Acknowledgements}

The authors would like to thank Jon Sandberg, Jian-Lin Mi, and Wu (Arlene) Zhang for support and advice. 